\title{
Pulsar Polarimetry - New Aspects at High Radio Frequencies
}

\author{
Alexis von Hoensbroech \\ Max-Planck-Institut für Radioastronomie, Auf dem Hügel 69, D-53121 \\ Bonn, Germany
}

\begin{abstract}
Pulsar polarimetry is an important tool for the investigation of the physical processes and the environment in the magnetosphere. However, the great variety of observed polarization states and the few obvious systematics hamper a straightforward interpretation. In this paper it is shown that the polarimetric features of pulsars are much more systematic at high radio frequencies. An analysis of 116 profiles at 4.85 $\mathrm{GHz}$ reveals a clear correlation between the degree of polarization and $\dot{E}$. Also evidence is shown for pulsars with a heavily increasing degree of circular polarization. Further, a basic concept of a simple magnetospheric propagation scenario is presented, which can account for a variety of polarimetric properties of pulsars.
\end{abstract}

\section{Observations}

General polarimetric features of pulsar radio emission include a high degree of mostly linear polarization, depolarization with increasing frequency, an " $\mathrm{S}$ "shaped polarization position angle (PPA) swing which seems to reflect the local magnetic field direction at or above the emitting region, and the occurrence of orthogonal polarization modes (OPM), i.e. jumps about $90^{\circ}$ in the PPA. In 1995 a new $4.85 \mathrm{GHz}$ receiver has been installed at the Effelsberg $100 \mathrm{~m}$ radio telescope. Its high sensitivity allowed the first large survey of pulsar polarization profiles in a frequency regime, which is significantly higher than the "classical" pulsar radio frequencies.

Two unexpected results originated among others (see von Hoensbroech et al 1998a) from this high radio frequency investigation: In contrast to lower frequencies, the degree of polarization is correlated to the rotational energy loss of the pulsar $\dot{E}$ (see Fig. 1). A subgroup of pulsars was found which shows a strongly increasing degree of circular polarization with frequency (see Fig. 2).

\section{Theoretical Approach}

A theoretical approach was applied which is based on birefringence of the propagating wave in the highly anisotropic magnetosphere This concept succeeds to qualitatively model the observations described above. For details, see von Hoensbroech et a.l. (1998b) and von Hoensbroech \& Lesch (1999). 

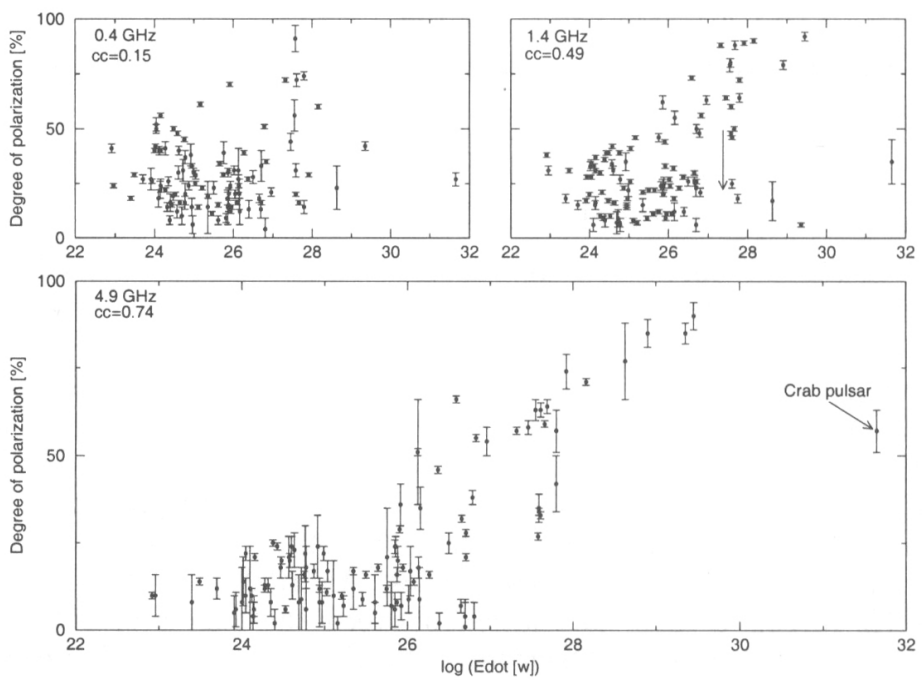

Figure 1. The degree of polarization versus the loss of rotational energy $\dot{E}$ for (nearly) the same set of 116 pulsars at $0.4,1.4$ and $4.9 \mathrm{GHz}$. Low frequency data from Gould \& Lyne (1998). cc denotes the correlation coefficient. The correlation becomes increasingly significant towards higher frequencies, suggesting that a low frequency randomization possibly disguises an underlying correlation.

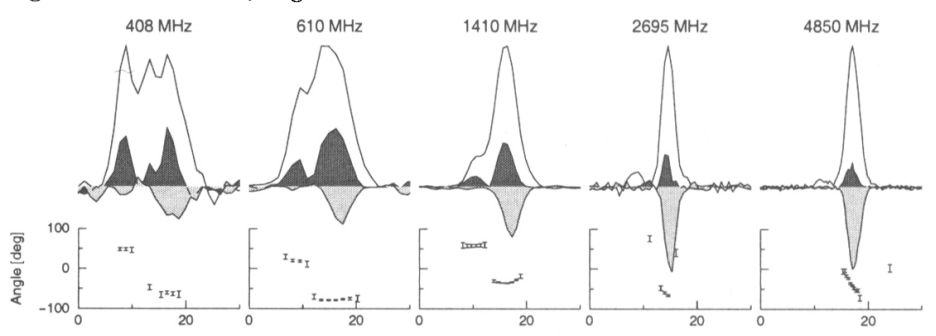

Figure 2. Example for a pulsar with increasing circular polarization (PSR B0144+59, un-, dark-, and light shaded areas represent total-, linear-, and circular polarization respectively). Presently three pulsars are known which show this smooth transition from linear to circular polarization.

Acknowledgements. The author especially thanks Richard Wielebinski and Harald Lesch for their support and active participation in this research, which was part of a PhD-Thesis (von Hoensbroech 1999).

\section{References}

Gould, Lyne, 1998, MNRAS, 301, 235

von Hoensbroech, 1999, PhD-Thesis, University of Bonn

von Hoensbroech, Lesch, 1999, A\&A, 342, L57

von Hoensbroech, Kijak, Krawczyk, 1998a, A\&A, 334, 571

von Hoensbroech, Lesch, Kunzl, 1998b, A\&A, 336, 209 